\title{
Visual feature integration and focused attention: Response competition from multiple distractor features
}

\author{
NILLI LAVIE \\ University College London, London, England
}

\begin{abstract}
Predictions from Treisman's feature integration theory of attention were tested in a variant of the response-competition paradigm. Subjects made choice responses to particular color-shape conjunctions (e.g., a purple cross vs. a green circle) while withholding their responses to the opposite conjunctions (i.e., a purple circle vs. a green cross). The results showed that compatibility effects were based on both distractor color and shape. For unattended distractors in preknown irrelevant positions, compatibility effects were equivalent for conjunctive distractors (e.g., a purple cross and a blue triangle) and for disjunctive distractors (e.g., a purple triangle and a blue cross). Manipulation of attention to the distractor positions resulted in larger compatibility effects from conjoined features. These results accord with Treisman's claim that correct conjunction information is unavailable under conditions of inattention, and they provide new information on response-competition effects from multiple features.
\end{abstract}

A fundamental issue for theories of attention and perception is the nature of unattended perception. Many theorists have argued that attention is such a crucial resource for full perception that perception is deficient without it. But what is the nature of this deficiency? What is the need in perception that attention must satisfy? The feature integration theory (FIT) of attention advanced by Treisman and her colleagues (e.g., Treisman, 1988; Treisman \& Gelade, 1980; Treisman \& Schmidt, 1982; Treisman, Sykes, \& Gelade, 1977) provides one clear suggestion. According to FIT, the visual features of unattended objects are registered separately, in independent representational maps that code values on various stimulus dimensions (e.g., sizes, colors, orientations). The conjunction of features into multidimensional objects in the scene (say, a small green backslash) is held to be mediated by focused attention. Thus, the selective operation of attention should result in a reduction or prevention of conjunctive coding for unattended objects, leaving the separate registration of their features relatively intact.

The issue of the role of attention in feature integration has been extensively studied over the last decade. The most common paradigms have involved visual search (e.g., Treisman \& Gelade, 1980), or "illusory conjunctions" (Treisman \& Schmidt, 1982). The conclusions regarding FIT that have been reached on the basis of these two paradigms remain somewhat controversial (see, e.g.,

I am grateful to Jon Driver, John Duncan, Anne Treisman, and Jehoshua Tsal for helpful discussions and comments on this research. I thank Sally Cox for her assistance in conducting the experiments and James Tresilian for his assistance with the figures. Correspondence should be addressed to N. Lavie, Department of Psychology, University College London, Gower Street, London WC1E 6BT, England (e-mail: n.lavie@ucl.ac.uk).
Duncan \& Humphreys, 1989, 1992; Prinzmetal, Henderson, \& Ivry, 1995; Treisman, 1991; Tsal, 1989; Wolfe, Cave, \& Franzel, 1989), as summarized briefly below.

The visual search paradigm initially provided support for FIT. Treisman et al. (1977) and Treisman and Gelade (1980) reported parallel search for feature targets and serial search for conjunctive targets, implying that only the latter requires focused attention. This pattern of results was subsequently replicated in several studies (see Treisman, 1988, for a review). However, numerous exceptions have accumulated as well. For instance, parallel set-size slopes have been reported for various conjunctive searches. Conjunction-search displays that involve the features of stereo disparity (e.g., Nakayama \& Silverman, 1986; Steinman, 1987) or motion (Driver \& McLeod, 1992; McLeod, Driver, \& Crisp, 1988) can allow particularly efficient filtering out of nontargets. Even search for conjunctions of color and shape features, the task that originally supported FIT, can be efficient in some cases. In particular, parallel search slopes are found when target-nontarget similarity and nontarget homogeneity are manipulated to provide a clearer segregation between target and nontarget perceptual groups (Duncan \& Humphreys, 1989; Wolfe, 1994; Wolfe et al., 1989).

These results have been accommodated by modifications to FIT in models that incorporate processes of attentional guidance and perceptual grouping based on feature processing outside focused attention (e.g., Cave \& Wolfe, 1990; Wolfe, 1994; Wolfe et al., 1989). These guided-search models account for cases of efficient search in terms of unattended feature-based processes, and so a special role for attention in the processing of conjunctions might still be retained theoretically, at least for inefficient searches (see, e.g., Treisman, 1991, 1992; Treisman \& Sato, 1990). 
In the present study, a new test of FIT is proposed in a focused attention paradigm, which does not involve any spatial search process. In this paradigm, targets are clearly distinct from distractors in terms of their spatial separation, with location certainty being maximal for both targets and distractors (target and distractors appear in fixed, preknown locations throughout, with the target always at fixation). Hence, any contribution of the factors discussed above that might influence search efficiency for targets in unknown locations (i.e., the factors of target-nontarget similarity, nontarget homogeneity, and preattentive guidance) should be minimized. In this way, I sought to emphasize any processing distinction there may be for conjunctions versus isolated features by making unattended processing of features less likely to contribute to attended processing of feature conjunctions by means of guidance (cf. Cave \& Wolfe, 1990). Since no search was required in my task, no guidance of search should arise.

The phenomenon of illusory conjunctions has provided the other main source of converging evidence for FIT. Treisman and Schmidt (1982) presented subjects with an array of multiple items and requested them to report first the primary target items (i.e., two digits that flanked a row of colored letters) and then all of the secondary target items (the colored letters) appearing in the array. Errors of feature miscombination (such as reporting a blue $\mathrm{X}$ and $\mathrm{a}$ brown $\mathrm{O}$ for a presented array of a brown $\mathrm{X}$ and a blue $\mathrm{O}$ ) were more frequent in the report of secondary items than were simple feature errors (such as reporting a red $O$ when red is part of the possible feature set but was not present in the current display). Since the secondary items were presumably less attended than were the primary targets, the illusory conjunctions in subject reports were taken to demonstrate that correct integration of features may not be available for objects from which attention has been diverted.

Illusory conjunctions have now been reported for a variety of tasks and items (see, e.g., Briand \& Klein, 1987; Fang \& Wu, 1989; Prinzmetal, 1981; Prinzmetal \& MillisWright, 1984; Prinzmetal et al., 1995; Treisman \& Paterson, 1984). However, as with the visual search paradigm, the interpretation of these phenomena as directly supporting FIT has not gone uncontested. Some illusory conjunction paradigms have been criticized for involving possible memory confounds and for failing to provide a direct test for the specific role of attention in feature perception (see, e.g., Tsal, 1989; Virzi \& Egeth, 1984). In addition, cuing manipulations intended to influence the extent to which the critical items were attended (see, e.g., Briand \& Klein, 1987; Prinzmetal, Presti, \& Posner, 1986; Tsal, Meiran, \& Lavie, 1994) have not always supported the original claims of FIT.

However, in some respects the illusory conjunction paradigm is less than ideal for providing a strong test of FIT's central claim about the processing of unattended items. This is because the "unattended" items in the illusory conjunction paradigms are in fact relevant to the task and usually must be explicitly reported. These fac- tors may have reduced the qualitative difference in perception that might have emerged if truly unattended and attended processing had been compared.

My brief summary of existing visual search and illusory conjunction findings suggests that further testing of FIT could usefully examine focused-attention situations that do not require any explicit report for the unattended features (cf. the illusory conjunction paradigm) or search for the target among spatially intermingled nontargets that are intended to be irrelevant but appear in possible target locations (as in visual search). A situation in which target location is known and distractors are entirely irrelevant to the required task should permit a stronger comparison of processing for attended items versus unattended items.

The present study develops such a test for FIT, using one of the classic paradigms of focused attention, namely the response-competition paradigm introduced by B. A. Eriksen and C. W. Eriksen (1974). This paradigm provides an indirect procedure for assessing the perception of unattended items by measuring their effects on the response times (RTs) to atiended items. Thus, unlike the illusory conjunction paradigm, the unattended items whose processing is examined never have to be reported. Unlike search tasks, the location of the relevant target item is known in advance, and subjects are required to focus on the target and completely ignore the irrelevant distractor items throughout. These unattended distractors can either be compatible or incompatible with the relevant response. Perceiving the identity of the unattended distractors should lead to longer RTs for targets that appear with incompatible rather than compatible distractors (B. A. Eriksen \& C. W. Eriksen, 1974).

Applying FIT to the response-competition paradigm leads to a strong (and somewhat counterintuitive) prediction for the pattern of compatibility effects from unattended items. Specifically, the effects from a pair of unattended distractors that include two critical features from the target set should not differ according to whether those target features are conjoined within one distractor object or disjoint between the two distractors.

Consider the following response-competition task, in which choice responses must be based on a combination of two features. Subjects make one response to a red $\mathrm{H}$ and another response to a green $\mathrm{U}$, while ignoring two flanking distractors. They can be forced to base their responses only, on the combination of two target features by requiring them to withhold responses on occasional no-go trials in which a green $\mathrm{H}$ or a red $\mathrm{U}$ appears as the target instead. In this situation, neither color nor shape alone is sufficient to specify one of the go responses.

On a go trial with a red $\mathrm{H}$ target, a green $\mathrm{U}$ can be presented as one of the two flankers to provide an incompatible distractor. On such a trial, one might expect interference because the alternative target (i.e., the green $U$ ) appears as a distractor and so may activate the incompatible response. Note that this is an example of a conjunctive distractor, since the two incompatible features 
(i.e., green color and U shape) are both presented within one common distractor item. The critical test of FIT lies in a comparison of the distractor effects for this conjunctive condition versus a disjunctive condition in which the two incompatible features are separated across the two distractor objects (e.g., for a red $\mathrm{H}$ target, a comparison of a green $U$ plus a blue $\mathrm{X}$ as distractors in the conjunctive condition, versus a green $\mathrm{X}$ and a blue $U$ as distractors in the disjunctive condition). On a strict version of FIT, equivalent effects are predicted for these disjunctive and conjunctive distractor conditions. The two conditions differ only in the specific conjunctions of features within the two distractors, and on FIT it is precisely this information about the correct feature combinations that should be unavailable for truly unattended objects.

This prediction of equal effects for disjunctive versus conjunctive distractors seems counterintuitive, because whereas the distractor object of a green $U$ in the conjunction condition exactly matches the currently incompatible target item, neither of the distractor objects in the disjunctive condition (a green $\mathrm{X}$ and a blue $\mathrm{U}$ ) matches this alternative target. Note, however, that this intuitive line of reasoning is based on comparing distractor objects (each treated as conjunctions) to the possible targets, rather than on considering merely the features that are present among the two distractors. If correct conjunction information is unavailable without attention, as FIT holds, then any activation of the incompatible response by the unattended distractors should be based only on the features present in the distractors, rather than on their exact conjunctions. In terms of the isolated features present, the conjunctive and disjunctive distractors are equally matched with the incompatible response. Thus, equivalent effects should be expected for conjunctive and disjunctive distractors if the processing of unattended items is feature based rather than conjunction based, as FIT claims.

In fact, little is known about whether responsecompetition effects can be based on integrated distractor objects or only on response activation via single features. Previous response-competition studies have typically varied only a single feature (usually simple aspects of shape $)^{1}$ for targets and distractors (e.g., B. A. Eriksen \& C. W. Eriksen, 1974; C. W. Eriksen \& B. A. Eriksen, 1979; Flowers, Warner, \& Polansky, 1979, Miller, 1991). It is thus uncertain whether previous compatibility effects were due to activation of responses by an integrated representation of distractor objects or to a responseactivation process that was merely feature based.

Thus, measuring response-competition effects in a situation in which choice responses are based on two target features, with these features either conjoint or disjoint in the flanking distractors, should lead to a better understanding of response-competition mechanisms. In particular, it should reveal whether the activation of responses that leads to response competition is based on single features, multiple features, or integrated objects. But most importantly, a strong novel test for FIT is provided. Results showing more response competition from conjunctive distractors than from disjunctive distractors would disconfirm FIT. Results showing equivalent effects would provide counterintuitive evidence consistent with FIT from a focused-attention paradigm that has not been previously used to test it.

\section{EXPERIMENT 1}

In order to make feature integration relevant for the task, the choice responses were based on particular conjunctions of colors and shapes in the target. Subjects were requested to make one response for a purple cross target and another for a green circle target and to withhold their responses if the target had the opposite combinations of color and shape (i.e., a purple circle or a green cross). On $25 \%$ of the trials, one of these no-go targets appeared. Their role was to ensure that responses could not be based on detection of just a single feature (e.g., merely detecting purple did not permit the purple cross response).

Two irrelevant distractors flanked the central target on each trial on either side. One shape feature and one color feature across these distractors could be either compatible or incompatible with the current target. Most importantly, either these two distractor features were conjoined (conjunctive condition) in one of the distractor items (e.g., a green circle) whereas the other distractor had two neutral features (e.g., a blue triangle), or the distractor features were disjoined (disjunctive condition) across the distractor items (e.g., a blue circle and a green triangle as distractors). Thus, the same distractor features appeared in both conjunctive and disjunctive conditions; only their combinations into distinct objects varied. Conjunctive or disjunctive incompatible trials would have a purple cross as target for the distractors described above, and compatible trials with these distractors would have a green circle as target. These various distractor conditions are illustrated in Figure 1.

Experiment 1 also included displays in which distractors were compatible (or incompatible) in color alone (feature-color condition) or in shape alone (featureshape). These conditions were included to ensure that any effects obtained from distractors in the conjunctive and disjunctive conditions were indeed reflective of processing for both shape and color (e.g., if processing of distractors involved just their shape, this would be revealed by equivalent distractor effects for the conjunctive, disjunctive, and feature-shape conditions, with no effect for the distractor in the feature-color condition). Finally, some displays had distractors with no features drawn from the possible target set (the neutral condition), but all four of the neutral features instead (i.e., a square and a triangle in brown or blue). This condition was intended to determine whether any effects from distractors with target features were due to facilitation from compatible features or to interference from incompatible features.

The following pattern of results is predicted if responsecompetition effects from unattended distractors are based on the separate processing of both shape and color features, but not on their integration into particular objects. 
Incompatible

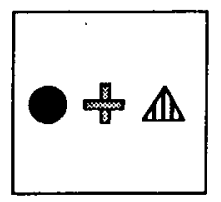

Conjunction

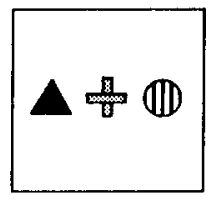

Disjunction

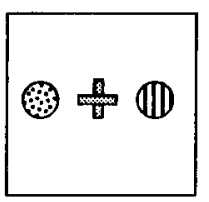

Shape

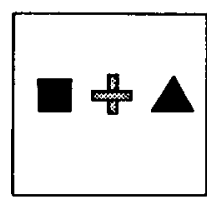

Color

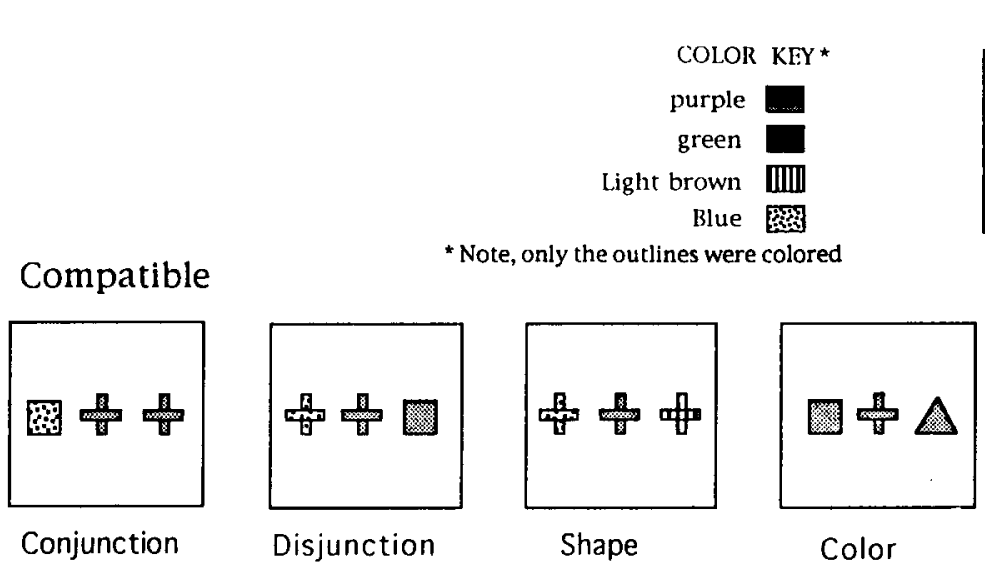

Neutral

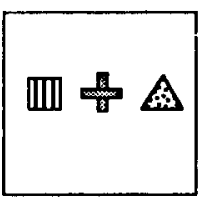

None

Figure 1. Examples of stimuli for Experiment 1.

Both the disjunctive and the conjunctive distractor conditions should show significant compatibility effects (i.e., response times should be slower with incompatible rather than compatible distractors), but there should be no significant difference between these conditions, since the stimuli differ only in how particular features are conjoined within individual unattended distractors. It is further required that any compatibility effects in the disjunctive and conjunctive conditions should be greater than in the feature-color and feature-shape conditions in order to ensure that they are not based on processing of just a single distractor feature (otherwise, the conjunctive vs. disjunctive issue would not arise).

\section{Method}

Subjects. The subjects were 12 undergraduates from the University of California, Berkeley, who participated in the experiment to fulfill a course requirement. All had normal or corrected-to-normal vision and reported no color blindness.

Apparatus. An IBM-PC-compatible computer attached to a VGA color monitor presented the stimuli and recorded the response latencies. The software used for creating and running the experiments was Micro Experimental Laboratory (MEL 1.0), sold by Psychology Software Tools (Schneider, 1988).

Design. There were two main types of trials: $75 \%$ of the trials were go trials and $25 \%$ of the trials were no-go trials. For the go trials, the target objects were either a purple cross or a green circle. For the no-go trials, the target objects had the opposite conjunctions of either a purple circle or a green cross. Hence the go/no-go task forced subjects to detect both the color and shape of the target. There were five conditions for the combinations of distractor features: Conjunctive and disjunctive conditions had the compatible (or incompatible) shape and color features conjoined within one distractor object or disjoined between two distractor objects, respectively. The feature-shape condition had just a compatible (or incompatible) shape feature repeated in both distractors, and the feature-color condition had just a compatible (or incompatible) color feature repeated in both distractors. The neutral condition had none of the target features in the distractors (Figure 1 shows examples of all these conditions). The critical distractor colors or shapes were repeated within each feature-color or feature-shape display in order to equate the total number of compatible (or incompatible) features appearing in the feature-color or feature-shape distractors as compared with the conjunctive or disjunctive conditions. Of course, this leaves a remaining difference between the feature-shape/ feature-color conditions and the disjunctive/conjunctive conditionsnamely, whether any distractor feature is repeated. This issue was addressed in Experiment 2. The no-go trials included all the same distractor conditions as the go trials. Conjunctive and disjunctive no-go distractor conditions consisted of the two features of one of the go targets arranged in a conjunctive or a disjunctive way, respectively. Note, however, that the "compatibility" of distractors with no-go targets was ambiguous. Consider, for example, a trial with a no-go target of a green cross. A conjunctive distractor of a green circle is incompatible in shape but compatible in color with this target; on the other hand, a conjunctive distractor of a purple cross is compatible in shape but incompatible in color. Hence, although no-go trials had all the conditions as the go trials, they were not analyzed in the same way. (The purpose of including all the distractor conditions in the no-go trials was mainly to avoid contingencies in the design, thus avoiding cuing the presence of a no-go target via its distractors.)

The composition of trials. There were eight trials for each subcategory of distractor type $\times$ compatibility per block (for example, eight with incompatible disjunctive distractors). Of these eight trials, six were go trials and two were no-go trials. Target identity was counterbalanced within each subcategory; 72 different displays allowed such a counterbalanced representation for all the conditions (eight trials for each subcategory $\times$ eight subcategories [four arrangements of distractor features $x$ two compatibility conditions], plus eight trials of the neutral condition).

Stimuli. All the shapes were colored outlines. The set of target features consisted of purple and green colors and cross and circle shapes. The set of possible distractor features consisted of these 
plus the neutral colors blue and light brown and the neutral shapes triangle and square. Each shape subtended $0.76^{\circ} \times 0.76^{\circ}$ of visual angle at a viewing distance of $60 \mathrm{~cm}$. The target and two flankers were positioned in a row, with $1.15^{\circ}$ of contour-to-contour distance between each shape. Each of the possible distractor features appeared equally often with each of the targets, in each of the compatibility conditions, and was equally likely to appear on the left or right side.

Procedure. Prior to each display, a light gray fixation dot appeared at the center of the display for $1 \mathrm{sec}$. This was immediately replaced by the target-plus-flankers display, which appeared for $150 \mathrm{msec}$. Subjects used two numerical keys on the right-hand side of the computer keyboard for their go responses and the space bar for the no-go responses (to initiate the next trial). They were required to press the $0 \mathrm{key}$ with their thumb for the go target of a purple cross and the 2 key with their index finger for the go target of a green circle, as fast as they could while avoiding errors. Feedback was given for both go and no-go errors by a $500-\mathrm{msec}$ computer tone. Subjects were also emphatically instructed to ignore the irrelevant distractors and were informed about the occasional presence of incompatible distractors and their possible detrimental effect on performance if these were not ignored. All the experimental conditions were presented in randomly intermixed fashion within each block of 72 trials. Trials with errors were not repeated. Each subject underwent nine blocks. The first block served as practice and its results were excluded from analysis. An intermission of 5 min was given after the first five blocks. The subjects initiated each block by pressing the space bar.

\section{Results and Discussion}

Accuracy in the go/no-go aspect of the task was important for confirming that subjects had indeed based their responses on processing both the color and shape features of the target correctly. Possible errors in the go/no-go task consisted of misses (withholding response for a go target) and false positives (responding to a nogo target). The average miss rate for go targets was $0.7 \%$. Preliminary inspection of the false-positive rates revealed that 1 subject had $26 \%$ false positives on the nogo trials. This subject's data were excluded from further analysis (but including them did not change the pattern of results). The average false-positive rate on no-go trials was $4 \%$. For most of the subjects ( 9 of the remaining 11 ), false positives were infrequent, ranging between $2 \%$ and $4 \%$. One subject had $9 \%$ false positives, and 1 had $10 \%$. Overall, then, it seems that most subjects complied with the demands of the task.

Figure 2 presents the median RTs for go trials averaged over the 11 analyzed subjects, as a function of distractor feature type (conjunctive, disjunctive, featureshape, feature-color, or none) and compatibility with the target (incompatible vs. compatible) for all except the none condition. RTs greater than $1.5 \mathrm{sec}$ were excluded from analysis. This eliminated only 25 correct trials across all subjects. Analyses were based on RT data, as there were very few errors in the choice responses with very little variation (see mean error rates in Figure 2).

The RT results are consistent with the hypothesis derived from FIT. According to FIT, only the separate features should be processed for unattended items, not their particular combinations into distinct objects. Hence, response competition from the present irrelevant and unattended distractors should not vary as a function of whether the features were conjoined or disjoined across a given pair of distractors. As can be seen in Figure 2, the

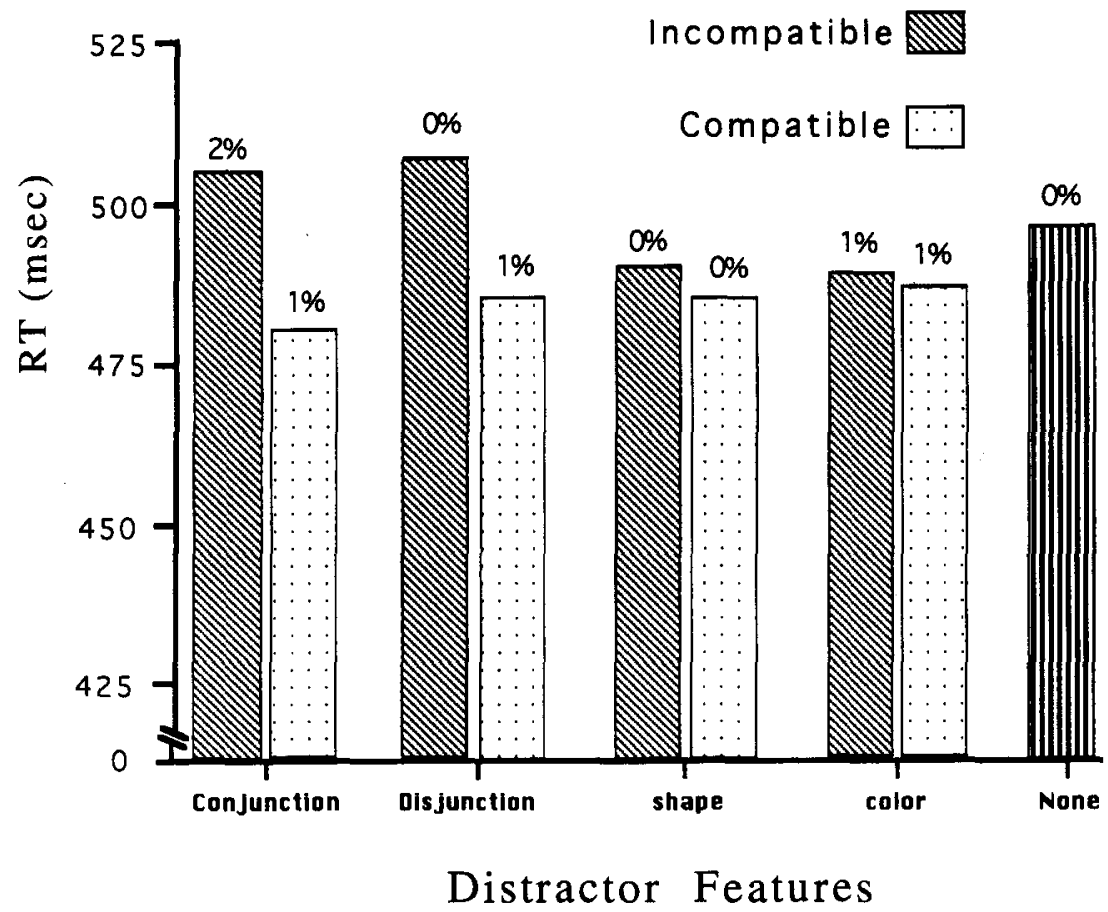

Figure 2. Experiment 1: Median choice response times (RTs) and percentage of errors as a function of distractor feature type and compatibility with the target. 
disjunctive and conjunctive conditions did indeed produce similar compatibility effects. Planned $F$ contrasts (testing for a $p$ value of .05) confirmed that RTs to the targets were significantly slower with incompatible than with compatible distractors in both these conditions $\left[F(1,10)=9.8, M S_{\mathrm{e}}=347\right.$ for the conjunctive condition; $F(1,10)=8.0, M S_{\mathrm{e}}=327$ for the disjunctive condition]. There was no difference $(F<1)$ between the compatibility effects for the disjunctive and conjunctive conditions. There was also no difference between the compatibility effects for the feature-color and feature-shape conditions $(F<1)$; in fact, neither of these produced a significant compatibility effect $(F<1$, for both). Finally, the compatibility effect obtained in the conjunctive/disjunctive conditions differed reliably from the null compatibility effect in the feature-color/feature-shape conditions $\left[F(1,10)=4.8, M S_{\mathrm{e}}=415\right]$.

Thus, we can conclude that when targets are defined by a particular conjunction of color and shape, the responsecompetition effect from unattended distractors requires that they have compatible (or incompatible) features in both color and shape, but it is not required that both these features appear conjoined within one distractor item. The compatibility effect that irrelevant and unattended distractors produce seems to be based on an unintegrated processing of their color and shape, regardless of the particular conjunctions that are present within the distractors. The counterintuitive result that response competition is no greater when the incompatible target appears as one distractor (conjunctive condition) than when its two features appear only across a pair of distractors (disjunctive condition) is just as predicted by FIT.

Further analyses compared compatible and incompatible distractors to the neutral condition in an effort to determine whether the compatibility effects observed were due to interference from incompatible distractors, facilitation from compatible distractors, or both. Since these were explorative post hoc comparisons, the Bonferroni adjusted $p$ value of .006 (i.e., .05/8) was used. No comparison with the neutral condition approached significance (nor did any reach the less conservative $p$ value of .05 ). Hence, the effects found in the comparisons of incompatible versus compatible distractors cannot be attributed solely to interference from incompatible distractors, nor solely to facilitation from compatible distractors. Instead, the overall compatibility effects seem to reflect the summation of both facilitation and interference components relative to the neutral condition (see Figure 2).

FIT did not make any specific prediction for potential effects in the feature-color or feature-shape conditions, given that the response mappings for targets in the present task depended on combinations of color and shape features. For present purposes, the feature-color and feature-shape conditions simply served to ensure that any effects in the disjunctive and conjunctive conditions were not based on the processing of just a single feature. Nonetheless, the absence of any compatibility effects in the feature-shape and feature-color conditions is an intriguing result, since many previous response-competition effects (see, e.g., Cohen \& Shoup, 1993; B. A. Eriksen \& C. W. Eriksen, 1974; C. W. Eriksen \& B. A. Eriksen, 1979; Flowers et al., 1979) were presumably based on single features.

The present null result in the single-feature conditions may have arisen because the target response had to be based on the conjunctive processing of both color and shape (go targets being a purple cross or a green circle, but not a green cross or a purple circle). As a result, any one of the features on its own (say, green) did not have an unequivocal association with one of the go targets (e.g., green circle) because it was also associated with one of the no-go targets that required a different response (e.g., green cross). Hence, the response association for any single feature was ambivalent in the present task; only the appearance of $t w o$ of the target features was unambiguously associated with a single response.

An alternative explanation for the present null result from single features would be that repetition of the distractor feature from the target set in the feature-color and feature-shape conditions (implemented in order to equate the total number of task-relevant features across distractor conditions) may have introduced some confounding variable. The appearance of displays that included repetition of shapes or colors was certainly different from that of any of the other displays (see Figure 1), and this may have caused some change in their processing. For example, the phenomenon of repetition blindness may have been involved only in these conditions (see Kanwisher, Driver, \& Machado, 1995). In Experiment 2, the repetition of the same features in the single-feature conditions was therefore avoided.

\section{EXPERIMENT 2}

The main aim of the second experiment was to replicate the results of Experiment 1, while generalizing it across different conjunctions of colors and shapes from those used in Experiment 1 to ensure that the results are not material specific. Thus, subjects were now requested to respond to a blue circle or a yellow triangle and to withhold their responses for the opposite combinations (i.e., blue triangle and yellow circle). Purple and green colors and cross and square shapes now served as neutral distractor features. The conditions of feature-color and feature-shape no longer involved any repetition of distractor features, since this may have been a confounding factor in the previous experiment, as discussed above. (Instead, a single feature from the target set appeared in only one of the two distractors for these two conditions.)

\section{Method}

Subjects and Apparatus. The subjects were 12 volunteers from the paid subjects pool of the MRC Applied Psychology Unit, Cambridge, who participated in exchange for $£ 5.50$. Their age range was from 18 to 33 years old. All subjects had normal or corrected-tonormal vision and reported no color blindness. The apparatus was equivalent to that for Experiment 1.

Stimuli and Procedure. The stimuli and procedure were identical to those of Experiment 1 except for the following changes. The 
targets for the go trials were a blue circle or a yellow triangle, and subjects were requested to press the 0 key for a yellow triangle and the 2 key for a blue circle. The neutral features consisted of purple, green, cross, and square. All the colored shapes were now filled in rather than outlines. In the feature-color and feature-shape conditions, there was now no repetition of distractor features. Instead, a single feature from the target set appeared in only one of the two distractors (the rest of three distractor features were neutral). Subjects participated in 13 blocks of 72 trials each. The first two blocks were discarded as practice.

\section{Results and Discussion}

Preliminary inspection revealed that 2 subjects had false-positive rates of $67 \%$ and $37 \%$ on the no-go trials. These subjects were excluded from further analyses (but including them did not change the pattern of results). The remaining subjects had an average rate of $11 \%$ false positives on no-go trials and of $0.09 \%$ misses on go trials.

Figure 3 presents the median RTs averaged over the 10 analyzed subjects as a function of distractor feature type (conjunctive, disjunctive, feature-shape, feature-color, or none) and compatibility with the target (incompatible or compatible) for all except the none condition. RTs greater than $1.5 \mathrm{sec}$ were excluded from analysis. This resulted in only 20 correct trials being discarded across all subjects. Errors in the choice responses were once again very infrequent (see mean error rates in Figure 3), and analyses were therefore based on the RT data. The results of Experiment 2 replicated the pattern obtained in Experiment 1. Planned $F$ comparisons of incompatible versus compatible distractors (again with a $p$ value of .05 ) revealed significant compatibility effects in both the con- junctive condition $\left[F(1,9)=10.6, M S_{\mathrm{e}}=203\right]$ and the disjunctive condition $\left[F(1,9)=9.4, M S_{\mathrm{e}}=136\right]$. There was no difference between these conjunctive and disjunctive compatibility effects $(F<1)$. Neither the featurecolor nor the feature-shape had any compatibility effect ( $F<1$ for each effect, and likewise for any difference between these null effects). The compatibility effect in the disjunctive/conjunctive conditions differed reliably from the null compatibility effect in the feature-color/featureshape conditions $\left[F(1,9)=4.9, M S_{\mathrm{e}}=304\right]$.

The replication of the null compatibility effect for singlefeature conditions (feature-color and feature-shape), now in the absence of any repeated distractor features, indicates that response-competition effects in the present task require the appearance of compatible/incompatible distractor features on both dimensions (even though it is not required that both features appear integrated within a common distractor object). This requirement for two features may arise because the present choice responses had to be based on the combination of both color and shape features, so any one feature on its own had only an ambiguous relationship to the choice response (see discussion of Experiment 1).

Further comparisons of compatible and incompatible distractors to the neutral condition were conducted using the Bonferroni adjusted $p$ value of $.006(.05 / 8)$, as before. No such comparison approached significance, suggesting that the compatibility effects in the conjunctive and disjunctive conditions reflected a summing of interference and facilitation effects rather than either alone. ${ }^{2}$

To sum up, the results of Experiment 2 replicated the results of Experiment 1, and generalized them across the

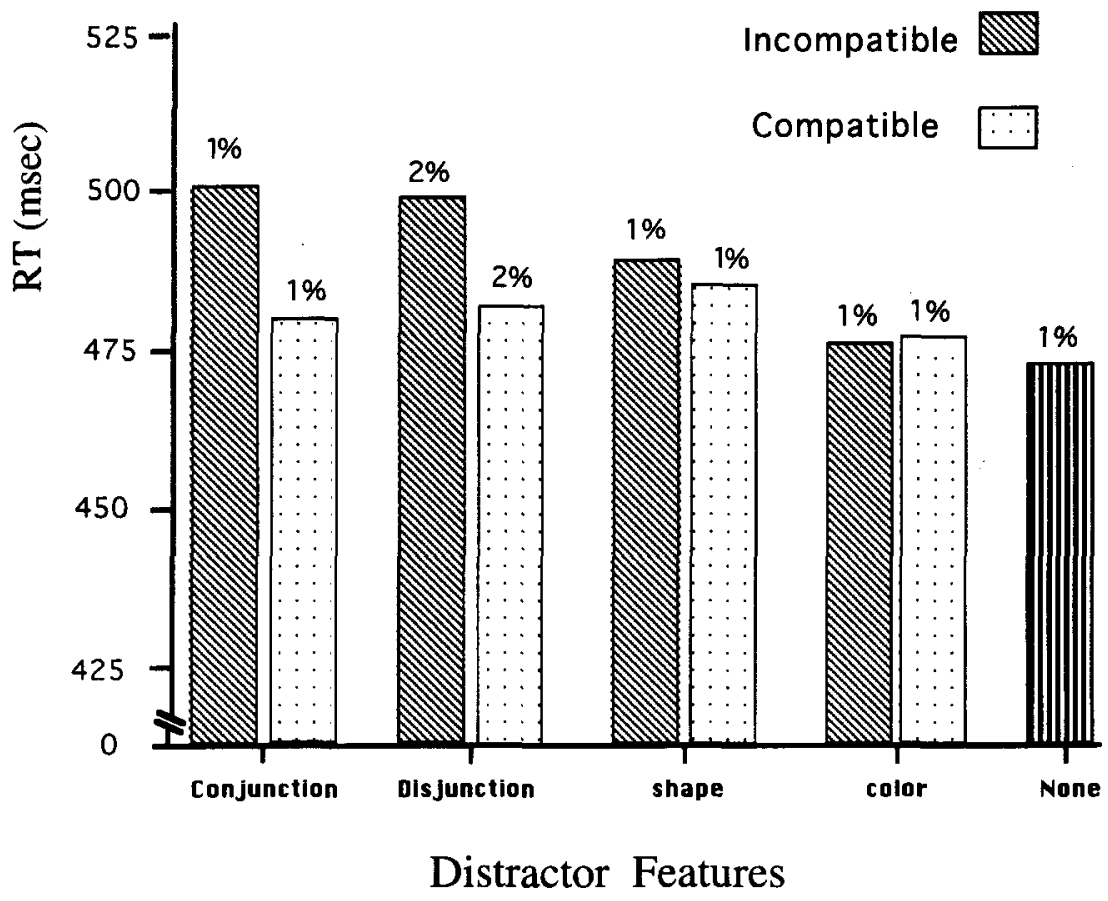

Figure 3. Experiment 2: Median choice response times (RTs) and percentage of errors as a function of distractor feature type and compatibility with the target. 
particular combinations of colors and shapes used and across whether features are repeated in the single-feature distractor conditions. I conclude that response-competition effects from unattended distractors in the present task are based on the separate processing of both their color and their shape features, but not on their conjunctions into individual distractor objects.

\section{EXPERIMENT 3}

The first two experiments revealed that responsecompetition effects were no greater for the conjunctive versus the disjunctive arrangement of distractor features. This is consistent with the prediction derived from FIT; namely, that correct conjunction information should not be available for unattended items. The purpose of the third experiment was to examine a complementary prediction from the role of attention in FIT; namely, that conjunction information should become available from attentive processing of the distractors. Testing this prediction may strengthen the claim that attention has a special role in feature integration. It may also allow us to confirm that the present response-competition measure has sufficient sensitivity to reveal any conjunctive processing for distractors.

The results so far might conceivably be attributed to some inherent inability of the present task to reveal any conjunction-based distractor effects. For instance, it might be that response-competition effects are insensitive to any integration of distractor features. Given that previous response-competition studies (e.g., B. A. Eriksen \& C. W. Eriksen, 1974; C. W. Eriksen \& B. A. Eriksen, 1979; Flowers et al., 1979) have used only feature-based choice responses, we cannot judge a priori whether response competition is an inherently feature-based rather than conjunction-based phenomenon.

Moreover, it might also be argued that despite our go/ no-go procedure, the task in Experiments 1 and 2 did not require true integration of features for the target, and thus compatibility effects could not be influenced by any true feature integration for the distractors. ${ }^{3}$ Although there are grounds for questioning this argument (see note 3 ), the general point that the response-competition measure might somehow have been insensitive to any feature integration for the distractors remains a possibility.

Experiment 3 was designed to examine whether the present task can reveal any conjunction-based responsecompetition effects and whether such effects are dependent on the allocation of spatial attention. It seemed particularly important to test whether distractor responsecompatibility effects can be conjunction based (rather than testing the nature of target processing in this task), as the novel aspect of the present paradigm was in the use of response-competition measures for distractor processing (see Treisman \& Schmidt, 1982, and Egeth \& Mordkoff, 1992, for the conjunctive processing of attended targets). In Experiment 3, attention to the distractors was manipulated while keeping the task otherwise the same as in the first two experiments. Choice responses were again based on processing two target features (subjects made go responses to distinguish a purple cross from a green circle and no-go responses to the opposite combinations of green cross and purple circle targets, as in Experiment 1). A target and two concurrent distractors were presented, as in the previous experiments. However, efficient focusing of attention upon just the target location was now prevented because the target was equally likely to appear at any one of the three possible positions in the array, rather than always appearing at the center, as before. Which item served as the target was now indicated by a low-contrast, thin bar cue that appeared simultaneously with the display of three items.

Thus, subjects could no longer focus attention on a known target location in advance, to the complete exclusion of distractors, because all positions were now potentially relevant. A distributed mode of attention across the positions of the array was now encouraged, making it likely that subjects would engage in processing all the items while the activation of the faint cue built up. A simultaneous but weak cue was used, rather than a post cue, in order to avoid any memory demands (see Tsal, 1989).

If attention facilitates the conjoining of features, as held by FIT, then conjunctive distractors should now produce larger effects than disjunctive distractors, since conjunction information should now be made available for distractors with the allocation of spatial attention. If, on the other hand, response competition is always based on the processing of separate features, equivalent effects should still be observed for the (now attended) conjunctive versus disjunctive distractors. Likewise, the results of Experiments 1 and 2 should be replicated once more if the present task is simply inherently insensitive to any feature conjunction for distractors, since the task was the same as for those previous experiments except for the change in the distribution of spatial attention.

\section{Method}

Subjects and Apparatus. The subjects were 12 volunteers from the paid subjects pool of the MRC Applied Psychology Unit, Cambridge, who participated in the experiment in exchange for $£ 5.50$. Their age range was from 18 to 33 years old. All subjects had normal or corrected-to-normal vision and reported no color blindness. The apparatus was the same as for Experiment 2.

Stimuli and Procedure. The stimuli and procedure were identical to those of Experiment l except for the following changes. The single-feature conditions (feature-color, feature-shape) did not include any repetition of distractor features. The neutral condition was eliminated, as Experiments 1 and 2 had found no reliable result in any comparison of compatible or incompatible distractors to neutral distractors. The target was now equally likely to appear in any one of three positions in a row at the center of the screen, with the distractors presented in the two other positions. A faint light gray horizontal bar of $0.05^{\circ}$ in width and $0.38^{\circ}$ in length was presented $0.4^{\circ}$ below the target to indicate its position in the current display. The three shapes and bar cue were now presented together for $200 \mathrm{msec}$ (this increased display time was implemented to produce a comparable accuracy rate with the previous studies, after pilot work indicated that a $150-\mathrm{msec}$ exposure duration resulted in some decrease in accuracy from Experiments 1 and 2). Each subject participated in 10 blocks of 96 trials each, preceded by 24 example tri- 
als. The example trials and the first block of 96 trials were discarded as practice.

\section{Results and Discussion}

Accuracy on the go/no-go task was quite high and comparable with the previous two experiments. The average false-positive rate on no-go trials was $8 \%$ (ranging from $1 \%$ to $22 \%$ ), and there were $1.6 \%$ misses on go trials.

Inspection of RTs revealed that 1 subject was very slow, with an average RT of $807 \mathrm{msec}$ for correct trials (over $2.5 S D$ from the average RT of 583 for the remaining subjects). This subject was excluded from any further analysis. RTs greater than $1.5 \mathrm{sec}$ were excluded from analysis. This resulted in 24 of the correct trials being discarded across all subjects. Figure 4 presents the median RTs averaged over the 11 analyzed subjects as a function of distractor feature type (conjunctive, disjunctive, featureshape, feature-color, or neutral) and compatibility with the target (incompatible vs. compatible). Errors in the choice responses were infrequent, with little variation across condition (see mean error rate in Figure 4), so analyses were based on the RT data.

A three-way within-subjects analysis of variance (ANOVA) was conducted with the factors of compatibility (incompatible, compatible), feature type (conjunctive, disjunctive, feature-color, and feature-shape), plus target position (center, left, or right). There was a main ef- fect of target position $[F(2,9)=7.5, p<.01]$, with faster responses to targets in the center (mean of $561 \mathrm{msec}$ ), than in the left $(594 \mathrm{msec})$ or right $(594 \mathrm{msec})$ positions, indicating the decrease in acuity with eccentricity. More importantly, there was a main effect of compatibility $[F(1,10)=17.7, p<.002]$ and a significant interaction between compatibility and feature type $[F(3,8)=14.15$, $p<.002]$. However, there was no interaction between target position and distractor compatibility $[F(2,9)=1.5$, $p>.27]$ nor between target position, compatibility, and feature type $[F(6,5)=2.2, p>.20]$. Thus, the compatibility effects and their interaction with feature type can be generalized across target positions. Planned $F$ contrasts (with a $p$ value of .05 ) found a significant compatibility effect in the conjunctive condition $\left[F(1,10)=29.2, M S_{\mathrm{e}}=\right.$ $394]$ and in the disjunctive condition $[F(1,10)=7.1$, $M S_{\mathrm{e}}=299$ ], but, as before, there was no compatibility effect in the feature-color or feature-shape conditions $(F<1$ in both cases). The primary interest of Experiment 3 was whether the conjunctive condition would now show a larger compatibility effect than that of the disjunctive condition. This comparison showed that the compatibility effect was indeed significantly larger in the conjunctive condition $\left[F(1,10)=5.3, M S_{\mathrm{e}}=351\right]$ for the first time (see also Figure 3)

Thus, the results of Experiment 3 demonstrate that conjunction information was now available for the distrac-

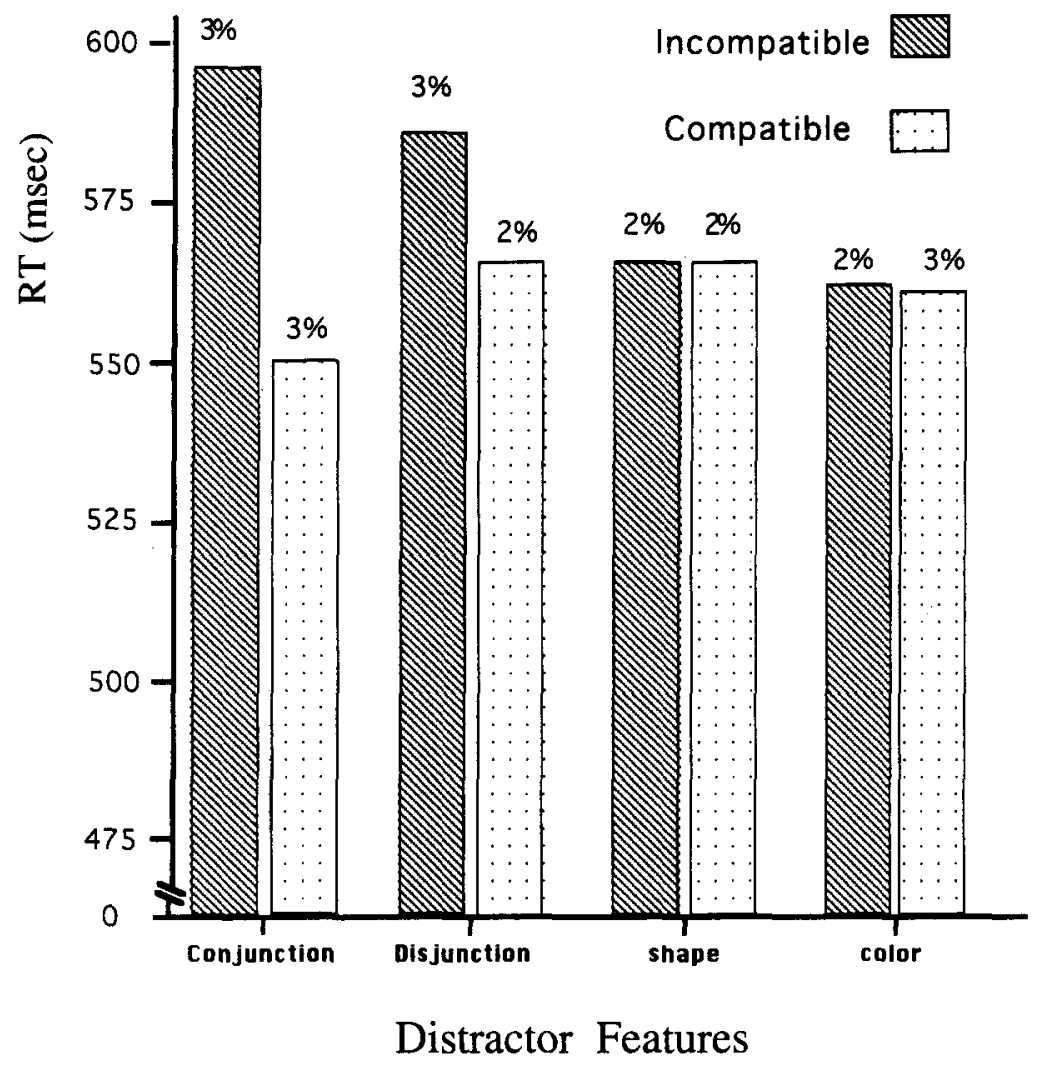

Figure 4. Experiment 3: Median choice response times (RTs) and percentage of errors as a function of distractor feature type and compatibility with the target. 
tors and could influence response competition accordingly because attention could no longer be focused on the target position in advance, to the exclusion of the distractors. Note that overall RTs were slower than in Experiments 1 and 2, even though Experiment 3 used exactly the same targets as those in Experiment 1 (i.e., purple cross and green circle). This seems to support the assumption that the reduced location certainty reduced the efficiency of target selection. Presumably attention was now allocated to all positions in the array, until focusing by the weak spatial cue built up.

Experiment 3 replicated the previous two studies' findings of no compatibility effect for the single-feature conditions (feature-color and feature-shape). Thus, it seems that this aspect of the results from all three experiments is a function of some inherent characteristics of the present task, regardless of the spatial allocation of attention (i.e., regardless of whether spatial allocation of attention is narrow, as in Experiments 1 and 2, or broad, as in Experiment 3 ). Presumably, the important inherent characteristic of the present task is that choice responses must be based on both the color and shape of the target so that single features have only ambiguous relationships with any response (see discussion of Experiment 1). As a result, compatible (or incompatible) distractor features are required on both dimensions to produce reliable responsecompetition effects. By contrast, previous studies have shown that when the choice responses can be based on just a single target feature (e.g., Cohen \& Shoup, 1993; Flowers et al., 1979), response competition can be found from single distractor features.

\section{GENERAL DISCUSSION}

The present study implemented a novel test for some of the most central and basic predictions of FIT, using one of the classic paradigms for studying focused attention, namely the response-competition paradigm (B. A. Eriksen \& C. W. Eriksen, 1974). The results of the first two experiments were consistent with FIT's prediction that the compatibility effects from unattended distractor features should not vary with whether the critical features are conjoined in one of the distractor objects or disjoined between them. RTs for attended targets that were defined by a conjunction of color and shape (e.g., green circle) and that appeared at a known central location were slowed down when the unattended distractors included both features from the target that was associated with the opposite response (e.g., a purple color and a cross shape) as compared with distractors that held both features of the current target (e.g., a green color and a circle). However, in Experiments 1 and 2, there was no difference between the effects that the two unattended incompatible or compatible features produced whether they were conjoined in one distractor object (e.g., a purple cross, plus the neutral distractor of a yellow triangle) or disjoined across two distractor objects (a purple triangle and a yellow cross).
This pattern of results indicates the perception of features along both color and shape dimensions for unattended distractors, but without any coding of the exact conjunction of those features into separate distractor objects. Note that the present task emphasized conjunction information, both for the processing of attended targets and in the instructions to ignore the potentially incompatible distractors. Nonetheless there was no hint of any greater compatibility effect from the conjunctive arrangement of the distractor features versus the disjunctive one, provided the task allowed efficient focusing of attention on the target to the exclusion of distractor positions (Experiments 1 and 2), by means of presenting target and distractors in fixed and well-separated positions.

In Experiment 3, the location certainty for target and distractors was reduced to prevent efficient focusing of attention and thus allow attention to be allocated to the distractors as well as the target. In this way, FIT's prediction that conjunction information should become available for attended distractors was tested. The target of Experiment 3 was equally likely in any of the three positions, with a weak simultaneous signal cuing its position. This was meant to prevent efficient selection of the target, thus inducing some processing for all the items in the array (distractors as well as the target) until the activation of the cue built up. The findings of Experiment 3 differed from those of Experiments 1 and 2 as a result of this positional uncertainty, demonstrating a larger compatibility effect for conjunctive than for disjunctive distractors. This confirms the prediction that conjunction information should be available with the attentive processing of distractors, and in so doing, demonstrates that the present responsecompetition task is sufficiently sensitive to reveal conjunction processing for the distractors when it does arise.

I conclude that the present results support the notion that perception of unattended objects results in registration of their visual features, but that attention is required for correctly combining these features into distinct objects. This accords with the original proposals of FIT (e.g., Treisman \& Gelade, 1980). Note that the present experiments could have falsified FIT had larger compatibility effects been found for conjunctive than for disjunctive unattended distractors in Experiments 1 and 2. The finding that a conjunctive distractor which is identical to the currently incompatible target produces no more response competition than does a pair of distractors which has its features disjoined across them provides a counterintuitive result that fits FIT. Furthermore, this finding is based on a well-established attention paradigm that has not been used to test FIT before.

\section{Implications for Theories of Visual \\ Feature Integration}

There seem to be two possible interpretations for the present pattern of results in terms of FIT in its original form (Treisman \& Gelade, 1980) and subsequent revisions to the theory (e.g., Treisman, 1988, 1991; Treisman \& Sato, 1990; Wolfe, 1994). The first and perhaps more 
straightforward interpretation suggests that the compatibility effects from the two distracting features of the unattended items (in Experiments 1 and 2) were based on an early stage of separate processing for both color and shape features, before any (correct or incorrect) conjunctive processing had occurred for the distractors. On this interpretation, the first two experiments would demonstrate that distractor effects can be based on such partial processing (i.e., at a level that includes both features, but not their conjunctions). Experiment 3 would then show that the distractor effects based on unattended feature processing were indeed somewhat incomplete, since compatibility effects were substantially boosted when attention was allocated to distractor processing (the compatibility effect was largest in this study; compare Figure 4 to Figures 2 and 3 ); moreover, attention to distractors selectively boosts just the compatibility effect from conjunctive distractors, indicating that attention specifically made conjunction information available for them for the first time.

The second possible interpretation invokes the phenomenon of illusory conjunctions for unattended items (Treisman \& Schmidt, 1982), as discussed in the introduction. Illusory conjunctions between unattended distractor features may have been responsible for the lack of any difference between conjunctive and disjunctive conditions in Experiments 1 and 2. On a proportion of trials, some of the disjunctive distractors may have been misperceived as conjunctive ones, and some of the conjunctive distractors as disjunctive ones, due to inattention for them. In fact, the amount of any such illusory conjunctions among the distractors in Experiments 1 and 2 would have to be roughly equal to that for "correct" combinations among the distractor features, since any smaller proportion of erroneous versus correct conjunctions would predict more substantial distractor effects with the conjunctive arrangement. This equivalent rate of incorrect and correct conjunctions for distractor features would, of course, arise by chance if no veridical conjunction information was available without attention (Treisman \& Schmidt, 1982). The allocation of attention to the distractors in Experiment 3 would increase the proportion of correct to erroneous conjunctions for them, thus producing more substantial effects from the conjunctive versus disjunctive distractor. Indeed, it may be worth noting that the effect was virtually doubled for the conjunctive distractor in Experiment 3 as compared with that for Experiments 1 and 2.

If the results of Experiments 1 and 2 really were due to illusory conjunctions, their occurrence must presumably have been perceptual, since no explicit report was required for the distractors and memory confounds should have been minimized (see previous criticisms of standard illusory conjunction paradigms, e.g., Tsal, 1989; Tsal et al., 1994; Virzi \& Egeth, 1984). It is impossible to choose between the two alternative interpretations presented above, which differ in terms of whether the compatibility effects were generated by independent coding of two distractor dimensions or by (random) conjunctions for those dimensions, on the basis of the present results. However, on either interpretation, the results still demonstrate that the correct integration of features is not necessary for obtaining response competition from unattended distractors. On both accounts, conjunction information would not be processed correctly for unattended distractor items; there would either be no conjunction, or this conjunction would be random for distractor features. Moreover, both accounts would also agree that allocating spatial attention to distractors (in Experiment 3) improves their conjunctive processing. Hence, both interpretations would support the role of spatial attention in feature conjunction that was originally proposed by FIT (Treisman \& Gelade, 1980).

This conclusion might appear to conflict with the conclusions from recent visual search studies, which have reported parallel search for some feature conjunctions (see McLeod et al., 1988; Nakayama \& Silverman, 1986; Wolfe et al., 1989) and so might be taken to imply that conjunction information can be available without attention. However, this apparent conflict can be resolved within the framework of existing models for visual search that have incorporated the recent search data. For instance, in the guided search model (Cave \& Wolfe, 1990; Wolfe, 1994; Wolfe et al., 1989), parallel conjunction search can arise whenever preattentive feature processing allows efficient grouping of all items sharing a nontarget feature. In such cases, groups of nontargets can be rejected or suppressed as a whole, with attention thereby guided toward likely candidates (or away from unlikely candidates) for the conjunctive target. Thus, on the guided search model, processing without focused attention may still involve just the parallel registration of separate features, in accordance with the original FIT and the present results. Nonetheless, by taking into account factors influencing search efficiency (e.g., the perceptual grouping of items and their heterogeneity; see also Duncan \& Humphreys, 1989), findings of parallel conjunction search as well as serial conjunction search can be accommodated by the guided search model, while still maintaining the assumption that conjunctive information is not available for unattended processing.

Note that the present experiments demonstrated processing of features for unattended items, as predicted by guided search, but using a paradigm quite different from the usual visual search paradigms in a situation that was originally developed by B. A. Eriksen and C. W. Eriksen (1974) specifically to yield a "nonsearch" task. In the focused attention situation of Experiments 1 and 2, location certainty was maximal, with the target appearing at a known and fixed position in the center of display, clearly segregated from the distractors in terms of spatial separation (with over $1^{\circ}$ of visual angle separating the target from distractors, e.g., C. W. Eriksen \& Hoffman, 1972). Hence, the first two experiments did not require any search for the target among the distractors, and the specific issue of feature integration could thus be distinguished 
from the more general issue of search efficiency. In Experiment 3 , more conjunction processing for the distractors was found under conditions of reduced location certainty for target and distractor positions. It was assumed that by preventing advance knowledge of target position, some spatial attention should now be allocated to the distractors, either by subjects adopting a wider setting of attention (e.g., C. W. Eriksen \& St. James, 1986) or by inducing some search for the target among the (now relevant) distractor positions. However, note that even if Experiment 3 did involve some process of visual search, which might be subject to similarity factors (Duncan \& Humphreys, 1989; Wolfe, 1994), the similarity between target and distractors, as well as within the distractors, remained exactly the same in Experiment 3 as in Experiments 1 and 2, yet very different results were found. Thus, similarity per se cannot account for the pattern of results found. Instead, the present experiments emphasize the allocation of spatial attention as an important determinant of feature integration for distractors. ${ }^{4}$

\section{Implications for Accounts of Response Competition}

The present experiments may also contribute to our understanding of the mechanisms behind response competition. Previous response-competition studies have typically used targets and distractors that vary in only a single feature (e.g., a choice response for $\mathrm{H}$ vs. U; see note 1 ), and effects of distractor compatibility have been obtained in many such single-feature tasks (see Lavie \& Tsal, 1994, for a recent review). Thus, the conditions under which response competition can be obtained from single-feature distractors are well known. However, the extent to which response competition can be based on multiple features (e.g., color in addition to shape), as well as how these features might combine to influence responses, was previously unknown. The present study certainly demonstrates that response competition can be based on processing multiple features from different dimensions (i.e., both color and shape). Compatibility effects were consistently obtained just for the conditions that had both target features in the distractors, with no such effects in the singlefeature conditions for all three experiments. Thus, the effects from the two-feature conditions could never be attributed to response activation from just a single feature.

Moreover, the two-feature effects could not be attributed to a simple process of summation for the effects from single features, either. Instead, the results consistently demonstrated overadditivity for the effects of conditions with both features present among the distractors, as compared with single-feature conditions. ${ }^{5}$ This may be attributed to the response mappings required in the present tasks to force the coding of both shape and color for the targets. The choice responses had to be based on combinations of the target features (e.g., a go response to a purple cross or green circle, but a no-go response to a green cross or purple circle). As a result of these mappings, any single feature (e.g., purple) had only equivocal response asso- ciations when taken in isolation, since it was linked with both a go response (e.g., to a purple cross) and a no-go response (e.g., to a purple circle). Hence, only the distractor conditions that involved both target features were unambiguously associated with a single response.

In effect, the imposed tasks forced adherence to an "and-gate" rule, on which a go response could be triggered only when both the features purple and cross were present in the target location; either feature on its own was insufficient. Interestingly, when this kind of rule had to be applied to the target events, response activation by distractors evidently also followed a similar and-gate principle, so that only distractors with both features could activate a response and so lead to compatibility effects. However, although the distractors had to involve both target features for producing any response competition, and although integration of features at the target location was required for making any correct target response (see note 3 ), integration of distractor features within a common distractor location was not necessary for activating an incompatible response (since equivalent effects were found for disjunctive as well as conjunctive distractors in Experiments 1 and 2). Thus, the and-gate operating on distractor features evidently did not require common physical location to form the "and" link. As discussed with a different terminology in the section above on implications for theories of visual feature integration, the and-gate for distractor processing might still have involved location, but with the position of distractor features being coded incorrectly due to illusory conjunctions. Alternatively, the and-gate for distractors may have taken a purely temporal form, so that two distractor features simply had to be present simultaneously to form an "and" link. On either account, the present results demonstrate for the first time that in a conjunctive task, a competing response can be activated by improper integration for unattended distractors, based on either just their temporal co-occurrence or on illusory conjunctions. Both these accounts demonstrate that correct integration of visual features requires spatial attention, and that response activation by unattended distractors can follow complex mapping rules involving multiple visual features.

\section{Conclusions}

These three experiments tested basic, falsifiable predictions from FIT, using an adaptation of the responsecompetition paradigm, which is a classic tool for studying unattended processing, but has never previously been applied to test FIT. Instead of falsifying FIT, the results produce counterintuitive evidence in support of its most basic claims, and provide new information on the level of complexity at which distractors can induce response competition. Evidently, the response-competition paradigm continues to prove useful for developing novel indirect measures of the extent of unattended processing, and the role played by attention in feature integration remains an intriguing question that can be approached afresh with such indirect measures. 


\section{REFERENCES}

Briand, K. A., \& Klein, R. M. (1987). Is Posner's "beam" the same as Treisman's "glue"? On the relation between visual orienting and feature integration theory. Journal of Experimental Psychology: Human Perception \& Performance, 13, 228-241.

CAVE, K. R., \& Wolfe, J. M. (1990). Modelling the role of parallel processing in visual search. Cognitive Psychology, 22, 225-271.

Cohen, A., \& Shoup, R. E. (1993). Orientation asymmetry in the flanker task. Perception \& Psychophysics, 53, 693-703.

DRIVER, J., \& MCLEOD, P. (1992). Reversing visual asymmetries with conjunctions of movement and orientation. Journal of Experimental Psychology: Human Perception \& Performance, 18, 22-33.

DunCaN, J., \& Humphreys, G. W. (1989). Visual search and stimulus similarity. Psychological Review, 96, 433-458.

DunCAN, J., \& Humphreys, G. W. (1992). Beyond the search surface: Visual search and attentional engagement. Journal of Experimental Psychology: Human Perception \& Performance, 18, 578-588.

EGETH, H. E., \& MORDKOFF, J. T. (1992, November). Object-based feature detection: A dissociation using the additivity diagnostic. Paper presented at the annual meeting of the Psychonomic Society, St. Louis.

ERIKSEN, B. A., \& ERIKSEN, C. W. (1974). Effects of noise letters upon the identification of a target letter in a nonsearch task. Perception \& Psychophysics, 16, 143-149.

ERIKSEN, C. W., \& ERIKSEN, B. A. (1979). Target redundancy in visual search: Do repetitions of the target within the display impair processing? Perception \& Psychophysics, 26, 195-205.

ERIKSEN, C. W., \& Hoffman, J. E. (1972). Temporal and spatial characteristics of selective encoding from visual displays. Perception \& Psychophysics, 12, 201-204.

ERIKSEN, C. W., \& Hoffman, J. E. (1973). The extent of processing of noise elements during selective encoding from visual displays. Perception \& Psychophysics, 14, 155-160.

ERIKSEN, C. W., \& ST. JAMES, J. D. (1986). Visual attention within and around the field of focal attention: A zoom lens model. Perception \& Psychophysics, 40, 225-240.

FANG, S. P., \& WU, D. (1989). Illusory conjunctions in the perception of Chinese characters. Journal of Experimental Psychology: Human Perception \& Performance, 15, 434-447.

Flowers, J. H., Warner, J. L., \& Polansky, M. L. (1979). Response and encoding factors in "ignoring" irrelevant information. Memory \& Cognition, 7, 86-94.

KanwiSher, N., Driver, J., \& MAChado, A. (1995). Repetition blindness in vision is modulated by attention to color and shape. Cognitive Psychology, 29, 303-337.

Lavie, N., \& Tsal, Y. (1994). Perceptual load as a major determinant of the locus of selection in visual attention. Perception \& Psychophysics, 56, 183-197.

MCLeOD, P., Driver, J., \& CRISP, J. (1988). Visual search for conjunctions of movement and form is parallel. Nature, 332, 154-155.

MiLleR, J. (1991). The flanker compatibility effect as a function of visual angle, attentional focus, visual transients, and perceptual load: A search for boundary conditions. Perception \& Psychophysics, 49, 270-288.

NaKayama, K., \& Silverman, G. H. (1986). Serial and parallel processing of visual feature conjunctions. Nature, 320, 264-265.

PrinzmeTal, W. (1981). Principles of feature integration in visual perception. Perception \& Psychophysics, 30, 330-340.

Prinzmetal, W., Henderson, D., \& Ivry, R. (1995). Loosening the constraints on illusory conjunctions: Assessing the roles of exposure duration and attention. Journal of Experimental Psychology: Human Perception \& Performance, 21, 1362-1375.

Prinzmetal, W., \& Millis-Wright, M. M. (1984). Cognitive and linguistic factors affect visual feature integration. Cognitive Psychology, 16, 305-340.

Prinzmetal, W., Presti, D. E., \& Posner, M. (1986). Does attention affect visual feature integration? Journal of Experimental Psychology: Human Perception \& Performance, 12, 361-369.

SCHNEIDER, W. (1988). Micro Experimental Laboratory: An integrated system for IBM PC compatibles. Behavior Research Methods, Instruments, \& Computers, 20, 206-217.
Steinman, S. B. (1987). Serial and parallel search in pattern vision. Perception, 16, 389-399.

Treisman, A. (1988). Features and objects: The fourteenth Bartlett Memorial Lecture. Quarterly Journal of Experimental Psychology, 40A, 201-237.

TreISMAN, A. (1991). Search, similarity, and integration of features between and within dimensions. Journal of Experimental Psychology: Human Perception \& Performance, 17, 652-676.

TREISMAN, A. (1992). Spreading suppressions or feature integration? A reply to Duncan and Humphreys (1992). Journal of Experimental Psychology: Human Perception \& Performance, 18, 589-593.

Treisman, A., \& Gelade, G. (1980). A feature-integration theory of attention. Cognitive Psychology, 12, 97-136.

Treisman, A., \& Paterson, R. (1984). Emergent features, attention, and object perception. Journal of Experimental Psychology: Human Perception \& Performance, 10, 12-31.

Treisman, A., \& Sato, S. (1990). Conjunction search revisited. Journal of Experimental Psychology: Human Perception \& Performance, 16, 459-478.

Treisman, A., \& Schmidt, H. (1982). Illusory conjunctions in the perception of objects. Cognitive Psychology, 14, 107-141.

Treisman, A., Sykes, M., \& Gelade, G. (1977). Selective attention and stimulus integration. In S. Dornic (Ed.), Attention and performance $V I$ (pp. 331-361). Hillsdale, NJ: Erlbaum.

TsaL, Y. (1989). Do illusory conjunctions support the feature integration theory: A critical review of theory and findings. Journal of Experimental Psychology: Human Perception \& Performance, 15, 394-400.

Tsal, Y., Meiran, N., \& Lavie, N. (1994). The role of attention in illusory conjunctions. Perception \& Psychophysics, 55, 350-358.

VIRZI, R. A., \& EGETH, H. E. (1984). Is meaning implicated in illusory conjunctions? Journal of Experimental Psychology: Human Perception \& Performance, 10, 573-580.

WOLFE, J. M. (1994). Guided Search 2.0: A revised model of visual search. Psychonomic Bulletin \& Review, 1, 202-238.

Wolfe, J. M., Cave, K. R., \& Franzel, S. L. (1989). Guided search: An alternative to the modified feature integration model for visual search. Journal of Experimental Psychology: Human Perception \& Performance, 15, 419-433.

\section{NOTES}

1. The shapes of alphanumeric characters are often treated as a single feature by FIT (e.g., Treisman \& Schmidt, 1982), presumably because the combinations of shape elements in any familiar letter or digit are highly overlearned.

2. The interference effects from incompatible distractors in the conjunctive and disjunctive conditions reached a nonadjusted level of significance at $p<.02$. This may hint that the main source of the observed compatibility effects was one of interference rather than facilitation. However, it is not clear whether the "neutral" none condition in this task was indeed neutral. Distractors in the neutral condition shared no features with any of the targets, so one might expect RTs for this condition to be slightly faster than for any of the other conditions that include task-relevant distracting features.

3. My thanks to Tram Neill for raising this point during the review process. He noted that even though the present go/no-go task forces both the color and shape of the target to be considered, perhaps this requirement does not entail a full integration of features at the target location, but merely a coding of their simultaneous occurrence. This might explain why the compatibility effects in Experiments 1 and 2 were dependent only on the simultaneous presence of two features from the target set among the distractors, rather than on their conjunction within just one distractor at a common location. However, there are several reasons to doubt this account. First, many aspects of the present task make it plausible that the targets were fully attended, so that no partial processing for them (e.g., reduced conjunction information) would be expected. The task involved minimal location uncertainty. All targets appeared at a known fixed location in the center of the display, with all distractors more than $1^{\circ}$ away. Previous work (e.g., C. W. Eriksen \& Hoffman, 1972, 1973) therefore suggests that subjects would be able to focus their spatial attention on the target to engage in its full process- 
ing. Second, Egeth and Mordkoff (1992) have recently compared conjunctive and disjunctive arrangements of target features. They concluded that subjects integrate features within target objects, even when the task requires only that the presence of two features be judged, regardless of their location. Third, and perhaps most importantly, full integration of target features (i.e., the coding that both features were present and that both were in the target position) was in fact required for achieving accurate performance in the present task. This requirement applied because almost half of the trials involved distractors with incompatible features. This would have led to many incorrect responses if judgments were based solely on the features present, rather than on their positions. Take, for example, a trial from Experiment 1 with a green circle for the target and the incompatible feature-color of purple for the distractors. If subjects did not integrate the color and shape fea- tures in the target location, they might have based their target judgment on the presence of purple, rather than green, along with the circle. This would have led subjects to make some no-go responses (appropriate to a purple circle) instead of the correct go responses, thus leading to many misses. However, misses were very infrequent in this study (less than $1 \%$ on average). By the same argument, choice go responses should also have been inaccurate, but accuracy was in fact very high, suggesting that target features were correctly integrated via their position.

4. My thanks to Art Kramer and an anonymous referee for raising these issues.

5. My thanks to Tram Neill for raising this point.

(Manuscript received September 2, 1994; revision accepted for publication June 13, 1996.) 